\title{
A Silicon-Polymer Heterostructure for Sensor Applications
}

\author{
J. M. G. Laranjeira, H. J. Khoury, \\ Departamento de Energia Nuclear, Universidade Federal de Pernambuco, \\ Cidade Universitária, 50740-540 Recife, Pernambuco, Brazil \\ W. M. de Azevedo, \\ Departamento de Química Fundamental, Universidade Federal de Pernambuco, \\ Cidade Universitária, 50670-901 Recife, Pernambuco, Brazil \\ E. A. de Vasconcelos, and E. F. da Silva Jr. \\ Departamento de Física, Universidade Federal de Pernambuco, \\ Cidade Universitária, 50670-901 Recife, Pernambuco, Brazil
}

Received on April 23, 2001

\begin{abstract}
We report the development and characterization of high quality polyaniline-silicon heterojunction diodes appropriated for use as gas and/or ionizing radiation sensors. Polyaniline thin films $40 \mathrm{~nm}$ thick are an active part of the junction structure, that presents excellent electrical characteristics, with rectifying ratio of 50,000 at \pm 1.0 Volt bias. The devices are very sensitive to $\gamma$-radiation up to 6,000 Gy and to gas moistures such ammonia, nitric acid and trichloroethylene. The sensitivity of the diodes is observed through shifts of the current-voltage (I-V) curves which can be easily monitored to provide a calibration curve of the sensor either as a radiation dosimeter or as a gas sensor for use in applications in environments demanding gas monitoring or radiation dosimetry.
\end{abstract}

In the last two decades conducting polymers emerged as a new class of material for use in advanced electronic device technologies[1, 2, 3, 4]. Their electrical conductivity can be changed from isolating to metallic by chemical or electrochemical doping. The electronic conductivity related to the redox state (doping level) of these polymers can be modulated by the interaction with various substances. Changes in resistance, current or electrochemical potential, give a straightforward sensor response of the studied phenomena.

Polyaniline is attractive for development of new devices because its electrical properties can be changed by oxidation or the main chain via protonation of the imine nitrogen backbone, keeping its thermal and environmental stability. Resistive polymer-based devices $[6,7,8]$ have demonstrated potential for applications as gas sensing by detecting vapor of a variety of gases. Early reports on electrochemically deposited polyaniline-silicon heterojunctions $[9,10]$ describe devices with low rectifying ratios (of about 50), and use relatively thick films, yielding devices with electrical characteristics that reveal and suggest the need for further improvement on device fabrication. A less studied subject is the use of conducting polymers for dosimetry of ionizing radiation. Few reports on polyaniline-based devices deals with conductivity changes under ionizing radiation exposure, but do not explain why the conductivity of polyaniline changes up to five orders of magnitude $[11,12]$, when emeraldine base interacts with ionizing radiation. Conflicting results reports either a conductivity increased [11] or decreases [12] after interaction with $\gamma$-radiation. Presently, there is no model that clearly explain results of the radiation effects in the physical and chemical properties of polyaniline or how ionizing radiation modify the conductivity of the polymers [13].

In this work we present the development of a new process to produce high quality polyaniline-silicon heterojunction diodes and examined the performance of the devices under exposure to ionizing radiation and gas vapor, by means of changes introduced to the device electrical characteristics.

The polyaniline-silicon heterojunctions were produced by spin-coating of polyaniline solution onto the silicon substrate. A $40 \mathrm{~nm}$ layer of polyaniline was deposited onto a n-type silicon (100) substrate of resistivity 1 $\Omega . c m$. All procedures were done in a clean room class 1000 and substrate cleaning followed a RCA standard process [14] with electronic grade chemicals. The polyaniline synthesis was carried out following the 
procedure described by of MacDiarmid et al [15]. The thickness of films were measured by a Rudolf Research Auto-EL IV Ellipsometer. Electrical contacts were deposited by vacuum thermal evaporation, with the device front contact made of a $400 \mathrm{~nm}$ gold film and the back contact of a $40 \mathrm{~nm}$ aluminum film. The devices have an effective area of $3.6 \times 10^{-3} \mathrm{~cm}^{2}$ and electrical characterization was carried out with a HP 4155A Semiconductor Parameter Analyzer. The ${ }^{60} \mathrm{Co}$ irradiation source was a 'gamma-cell' Cobalt irradiator, from Radionics Labs at a dose rate of $0.2742 \mathrm{~Gy} / \mathrm{min}$.

Optical properties of polyaniline solution and thin films were previously studied [14] to assure the doping state of the polymer. UV-visible (solution) and FTIR (film) spectra indicated that the polymer film is in the doped state[14, 16, 17], therefore forming a p-n heterojunction with silicon. The synthesis method and spin-coating of polyaniline on silicon using organic acids has demonstrated to yield excellent reproducibility of the heterojunction electrical characteristics. For the devices fabricated we obtain reverse breakdown voltages of -70 Volts, reverse saturation current of $6 \mathrm{nA}$, rectifying ratio of 50,000 (at 1 Volt) and ideality factor of 1.004 . These parameters values are important for sensor applications of the diode for spectrometry or dosimetry of high energy ionizing radiation.

Figure 1 shows the behaviour of the I-V characteristics of a diode before and after exposure to 3,000 Gy of $\gamma$-radiation. The main radiation effect on the device characteristic is a parallel shift in both forward and reverse bias regions, with no further sign of device degradation. Figure 2 presents in greater detail the relative changes $\left(\Delta \mathrm{I} / \mathrm{I}_{o}\right)$ in forward and reverse currents for $\gamma$-radiation doses in the range of 0-6,000 Gy. In reversed bias configuration (curve (a)) there is a continuous increase in current, linear with dose, and the relative changes $\Delta \mathrm{I} / \mathrm{I}_{o}$ reaches up to $1,400 \%$. This is a consequence of ionizing radiation effects associated to electron-hole pair generation due to interaction of the radiation with the polymer structure. In contrast, in the forward bias regime, there is a transient very rapid increase in current and after a small initial dose the relative current changes reaches $800 \%$. From there after, the behavior with dose is toward a current reduction yielding the linear behavior as shown in curve (b) of Figure 2. The effect of radiation on the heterojunction conductivity is attributed to changes in oxidation state of polyaniline during irradiation with $\gamma$-radiation[13], but the precise mechanisms are under study. These results suggest that measurements requiring low doses (under 2,000 Gy) and high sensitivity could be done in the reversed bias mode, while higher doses (over 2,000 Gy) could be monitored in the forward bias condition, at expenses of some loss in sensitivity.

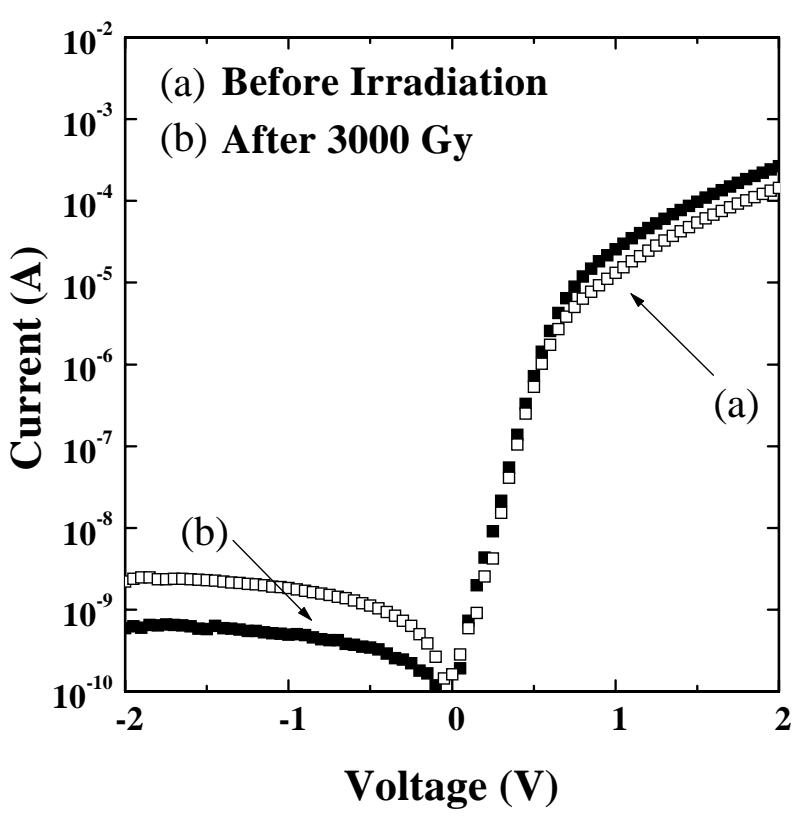

Figure 1. I-V characteristics of the heterojunction diode prior and after exposure to 3,000 Gy of $\gamma$-radiation.

The potential of using the heterojunction as a gas sensor is illustrated in Figure 3. The diodes were exposed to different gases and its electrical response measured as a function of exposure time. Curve (a) represents the virgin diode, prior to vapor exposure. Curve (b) is the resulting $\mathrm{I}-\mathrm{V}$ characteristic after one minute exposure to ammonia. The forward characteristic is strongly shift positively relative to curve $(\mathrm{a})(+\Delta \mathrm{V})$, and this behavior is found to be linear within the exposure time interval of 0-5 minutes. Larger exposure time leads to larger positive voltage shifts, and we observed that the device response is highly sensitive and fast. Electron donating gases like ammonia interacts with the polymer and reduces the carrier density of polyaniline film, towards a resistance increase. The responses of the device to trichloroethylene for 5 minutes (curve(c)) and nitric acid for 1 minute (curve (d)) are also presented. The responses are quite different from ammonia. The forward characteristic shifts negatively relative to curve $(\mathrm{a})(-\Delta \mathrm{V})$ as a function of exposure time, however the linear dependence with exposure time remains unchanged. All devices also present a high sensitivity, and relative current changes reaches $54 \%, 68$ $\%$ and $17 \%$ respectively for ammonia ( 1 minute), nitric acid ( 1 minute) and trichloroethylene ( 5 minutes), at 2 Volts bias. Here, protonic acids increase the carrier density of the polyaniline thin film and therefore decrease the resistance through the heterojunction. The polymer interaction with trichloroethylene vapors can be explained due to slow decomposition of trichloroethylene with subsequent formation of hydrocloridic acid under light action and in presence of the moisture. We are presently analyzing the sensor response to organic vapor compositions such as acetone, methanol, and a 
variety of other gases associated with combustion emission to unveil further applications of this device to contamination and environmental emission control.

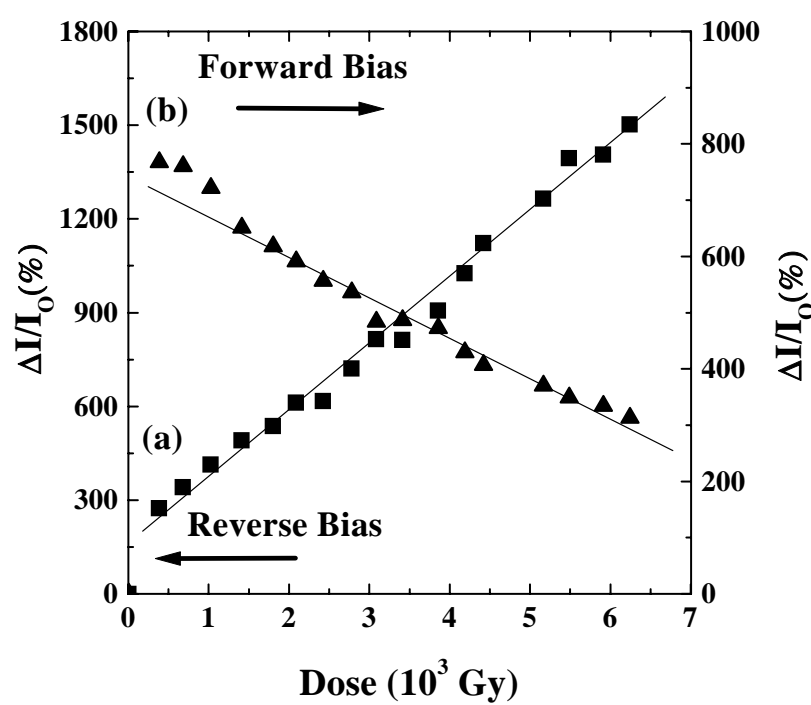

Figure 2. (a)Reverse and (b)Forward relative changes in current under $\gamma$-radiation doses in the range 0-6,000 Gy

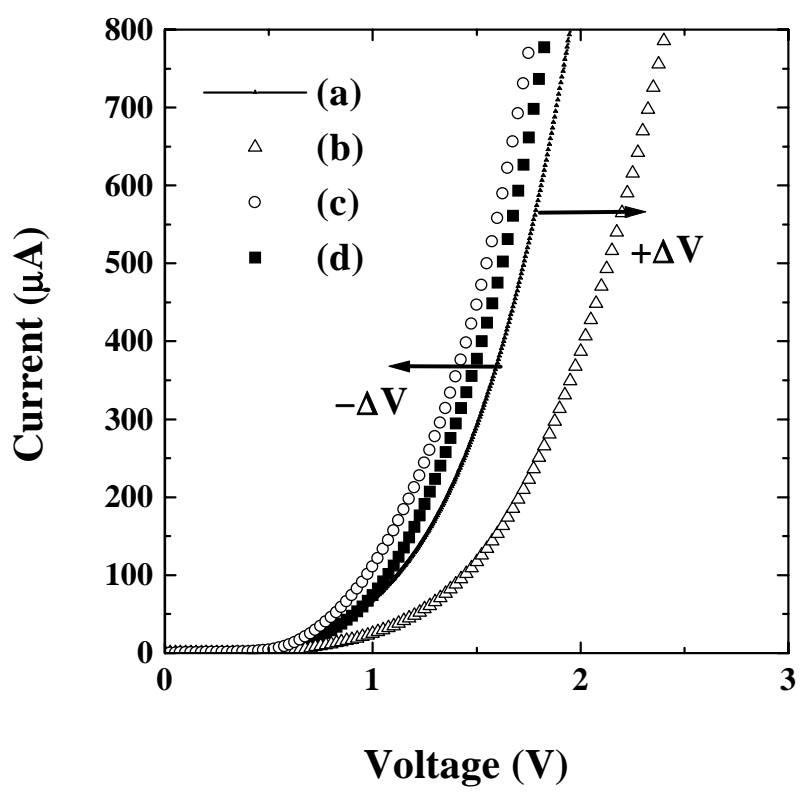

Figure 3. Current-voltage curves (a) prior to exposure, and after exposure to (b) ammonia for 1 minute, (c) trichoroethylene for 5 minutes and (d) nitric acid for 1 minute.

In summary, a process to produce high quality polyaniline-silicon heterostructure diodes has been developed. The devices present excellent electrical characteristics, with apparent barrier high of 0.58 volts, reverse bias current of $6 \mathrm{nA}$, ideality factor of 1.004 and rectifying ratio of 50,000 at \pm 1.0 Volt. Exposure to $\gamma$-radiation alters the forward and reverse current heterojunction characteristics and a linear behavior with radiation doses in the range of 0-6,000 Gy indicate the device suitability for dosimetry in the medical physics area. Exposing the diodes to gas moisture of ammonia, nitric acid, and trichloroethylene also alters linearly the diode forward characteristics. The behavior observed in the forward and reverse characteristics of the diodes are associated to changes of electrical conductivity of the polyaniline thin film and/or gas vapor adsorption. The results strongly suggest this type of heterojunction as a promising device structure for remote sensing of a variety of gases and high energy ionizing radiation.

\section{Acknowledgments}

The authors are thankful to the financial support received during the development of this work from $\mathrm{CNPq}$, PADCT/FINEP under contract \# 77.97.1120.00, and CTPETRO/FINEP under contract \# 65.00.02.80.00.

\section{References}

[1] H. Tomazawa, D. Braun, S. Phillips, A. J. Heeger and A. H. Kroemer, Synth. Met., 22, 63 (1987).

[2] H. Koezuka, and A. Tsumura, Synth. Met., 28, C753 (1989).

[3] G. Gustafsson, G. M. Treacy, Y. Cao and A. J. Heeger, Synth. Met., 55-57, 4123 (1993).

[4] J. C. Chiang A. G. MacDiarmid, Synth. Met., 13, 193 (1986).

[5] J. W. Gardner, P. N. Bartlett K. F. E. Pratt, IEE Proceedings-Circuits Devices and Systems, 142, 321 (1995).

[6] A. Boyle, E. Genis and M. Lapkowski, Synth. Met., 28, C769 (1989).

[7] C. Conn, S. Sestak, A. T. Baker and J. Unsworth, Electroanalysis, 10, 1137 (1998).

[8] E. W. Paul, A. J. Ricco, M. S. Wrighton and Y. Nishioka, J. Phys. Chem., 89, 1441 (1985).

[9] Y. Renkuan, Y. Hong, Z. Zheng, Z. Youdou and W. Yongan, Synth. Met., 41-43, 731 (1991).

[10] Y. Renkuan, L. Yuxue, Y. Hong, W. Yongbin, Z. Xiangqin and X. Jian, Synth. Met., 55-57, 4087 (1993).

[11] Q. Yao, L. Liu and C. Li, Polymer Bull., 31, 601 (1993).

[12] M. Wolszczak, J. Kroh and M. M. Abdel-Hamid, Radiat. Phys. Chem., 45, 71 (1995).

[13] W. M. de Azevedo, A. P. Lima, E. S. Araújo, Rad. Prot. Dosimetry, 84, 1 (1999).

[14] J. M. G. Laranjeira, H. J. Khoury, W. M. de Azevedo and E. F. da Silva Jr., Annals of the $V$ Encontro $\mathrm{Na}$ cional de Aplicações Nucleares, 5, N.1, p.154-158 (2000).

[15] A. G. MacDiarmid, J. C. Chang, A. F. Richter, N. L. D. Somasiri and J. Epstein, in Conducting Polymers, Ed. L. Alcacer, Reidel Publ. Co., 105 (1987).

[16] M. K. Ram, G. Mascetti, S. Paddeu, E. Maccioni and C. Nicolini, Synth. Met., 89, 63 (1997).

[17] J. Tang, X. Jing, B. Warig and F. Wang, Synth. Met., 24, 231 (1988). 